\title{
Balantidium coli
}

National Cancer Institute

\section{Source}

National Cancer Institute. Balantidium coli. NCI Thesaurus. Code C122246.

A species of ciliated intestinal protozoan parasites in the family Balantiididae. Infection occurs by ingestion of a cyst, with the motile trophozoite residing in the large intestine. B. coli is the largest protozoan that infects humans. 